\title{
Hiperinsulinismo endógeno: revisão e seguimento de 24 casos
}

\section{Endogenous hyperinsulinism: review and follow-up of 24 cases}

João S. Felício', Carlliane P. Martins', Mauro Semer', Larissa Kalinin', Marcia Nery', Marcel C. C. Machado', Bernardo Liberman'

\section{SUMÁRIO}

Em decorrência do hiperinsulinismo endógeno ( $\mathrm{HHE}$ ), a hipoglicemia é diagnosticada em um indivíduo sintomático com níveis baixos de glicose plasmática, concomitante a valores elevados de insulina plasmática e peptídeo-C. Entre as causas de HHE, estão as doenças das células- $\beta$ das ilhotas pancreáticas, o uso de secretagogos e a hipoglicemia autoimune. Neste artigo de revisão, estudamos 24 pacientes com hipoglicemia decorrente de hiperinsulinismo endógeno com a finalidade de descrever os aspectos de diagnóstico e tratamento. Nosso estudo mostrou que, após a realização do teste de jejum de 12h (minijejum) em três dias diferentes, todos os pacientes preencheram os critérios diagnósticos de HHE. Adicionalmente, encontramos que 11 dos 12 pacientes $(91,7 \%)$ que realizaram o teste do glucagon apresentaram níveis de glicose no tempo 120 minutos menores que $50 \mathrm{mg} / \mathrm{dL}$ e inferiores ao valor basal. 0 teste do minijejum (3 amostras) e o teste do glucagon poderiam ser úteis para evitar a realização do jejum prolongado no diagnóstico do hiperinsulinismo endógeno. Arq Bras Endocrinol Metab. 2012;56(2):83-95

Descritores

Hiperinsulinismo; insulinoma; hipoglicemia; pâncreas

\section{SUMMARY}

Hypoglycemia due to endogenous hyperinsulinism (EH) is diagnosed in a symptomatic patient with low levels of plasma glucose concomitant with elevated plasma insulin and C-peptide. Causes of EH are pancreatic islet-cells disease, use of insulin secretagogues, and autoimmune hypoglycemia. In this review, the authors studied 24 patients with hypoglycemia due to endogenous hyperinsulinism in order to describe aspects of diagnosis and treatment. Our study demonstrated that after 12 hours of fasting (mini-fasting test; at least three samples), all patients presented the diagnostic criteria for EH. Additionally, we found that 11 of 12 patients $(91.7 \%)$ who underwent glucagon test achieved glucose levels less than $50 \mathrm{mg} / \mathrm{dL}$ and below baseline after 120 minutes. Mini-fasting ( 3 samples) and glucagon test may be useful to prevent prolonged fasting test to clarify the diagnosis of endogenous hyperinsulinism. Arq Bras Endocrinol Metab. 2012;56(2):83-95

\section{Keywords}

Hyperinsulinism; insulinoma; hypoglycemia; pancreas
1 Serviço de Endocrinologia do Hospital Brigadeiro (SUS - São Paulo), Serviço de Endocrinologia da Universidade Federal do Pará (UFPA) e Serviço de Cirurgia das Vias Biliares do Hospital das Clínicas da Faculdade de Medicina da Universidade de São Paulo (HCFMUSP), São Paulo, SP, Brasil

Correspondência para: Carlliane P. Martins carllianepmartins@hotmail.com

Recebido em 17/Jan/2011 Aceito em 31/Jan/2012

\section{INTRODUÇÃO}

A glicose é um substrato metabólico fundamental para o cérebro, sendo a prevenção e a correção da hipoglicemia fundamentais para a manutenção da vida (1). A hipoglicemia pode ser dividida em pós-prandial (até 5 h após a refeição) e de jejum e seu diagnóstico se baseia na presença da tríade de Whipple (glicose plasmática baixa, sintomas compatíveis com hipoglicemia e melhora dos sintomas após o aumento nas concentrações de glicose), cuja presença deve impelir à análise de suas potenciais causas, sendo essa investigação fortemente recomendada $(2,3)$. Entretanto, o diagnóstico é praticamente inequívoco mesmo na ausência de sintomas se os valores de glicose plasmática estiverem abaixo de: $50 \mathrm{mg} / \mathrm{dL}$ na idade adulta e na adolescência $(4,5), 40 \mathrm{mg} / \mathrm{dL}$ na criança pré-púbere $(6), 30 \mathrm{mg} / \mathrm{dL}$ no neonato a termo e $20 \mathrm{mg} / \mathrm{dL}$ no neonato prematuro e/ou pequeno para a idade gestacional (7). En- 
tretanto, vale considerar que $10 \%$ dos indivíduos normais têm valores de glicose plasmática menores que 50 $\mathrm{mg} / \mathrm{dL}$ durante uma prova de tolerância oral à glicose de 5 horas (8) e que mulheres aparentemente normais podem ter valores de glicose plasmática menores de 40 $\mathrm{mg} / \mathrm{dL}$ durante o jejum prolongado $(3,9,10)$.

A hipoglicemia, em decorrência de hiperinsulinismo endógeno (HHE), é diagnosticada em um indivíduo com níveis baixos de glicose plasmática, concomitante a valores de insulina plasmática $>3 \mathrm{uU} / \mathrm{ml}$ e peptídeo-C $>0,6 \mathrm{ng} / \mathrm{ml}$, segundo recentes diretrizes da Endocrine Society (3). Entre as causas de HHE, estão as doenças das células- $\beta$ das ilhotas pancreáticas (adenomas solitários e múltiplos, hiperplasia e carcinoma), uso de secretagogos das células- $\beta$ (sulfonilureias) e a hipoglicemia autoimune (anticorpos contra insulina e seus receptores). O diagnóstico precoce dessas condições é de fundamental importância devido, principalmente, ao potencial tanto de letalidade quanto de cura da hipoglicemia. Os tumores secretores de insulina das ilhotas pancreáticas, os insulinomas, são raros, mas representam a principal causa de HHE em adultos, ocorrendo numa incidência de 4 a cada 1.000 .000 de indivíduos (11). Eles são responsáveis por $93,8 \%$ dos casos de hipoglicemia devido ao hiperinsulinismo endógeno, enquanto $6,2 \%$ são causados por doença difusa das células- $\beta$ (hiperplasia ou nesidioblastose, hipertrofia $\mathrm{e}$ microadenomatose) (12). Cerca de $80 \%$ dos insulinomas ocorrem entre 20 e 60 anos, com a idade média ao diagnóstico em torno de 47 anos. Frequentemente são lesões únicas, exceto quando se associam com neoplasia endócrina múltipla do tipo I (NEM-I: hiperparatireoidismo primário, adenoma hipofisário e tumores das ilhotas pancreáticas), o que ocorre em $8 \%$ dos casos, sendo os múltiplos tumores a regra nessa condição. Apenas 6\% dos insulinomas são malignos (11). Relatamos neste estudo a experiência com HHE do Serviço de Endocrinologia do Hospital Brigadeiro (SUS-SP), do Serviço de Endocrinologia da Universidade Federal do Pará (UFPA) e do Serviço de Cirurgia das Vias Biliares do Hospital das Clínicas da Faculdade de Medicina da Universidade de São Paulo (HCFMUSP).

\section{PACIENTES E MÉTODOS}

Foram estudados 24 pacientes com HHE, sendo 19 por insulinoma, três por nesidioblastose, e dois com histopatologia não conhecida (pacientes não submetidos à cirurgia). Entre os portadores de insulinoma, a idade média foi de 35 anos (variando de 16 a 68 anos) e o índice de massa corpórea (IMC) teve média de 24,6 $\mathrm{kg} / \mathrm{m}^{2}$ (variando de 13,6 a 34,8 ), sendo nove homens $(47,37 \%)$ e 10 mulheres $(52,63 \%)$. Os pacientes com nesidioblastose tinham idades de 2 anos e 6 meses e 1 ano, sendo um do sexo masculino e outro do sexo feminino, respectivamente (Tabela 1). Uma paciente de 38 anos tinha nesidioblastose com início da manifestação na idade adulta. Entre os 19 portadores de insulinoma, $6(31,58 \%)$ tinham NEM-I.

O diagnóstico de HHE foi baseado em dosagens plasmáticas de glicose, insulina e peptídeo-C após jejum durante a noite (12h) e jejum prolongado. As amostras após jejum durante a noite foram colhidas em pelo menos três oportunidades, sendo considerada a com menor nível de glicose plasmática. Adicionalmente, foram realizados os testes do Glucagon e tolerância oral à glicose (TOTG).

O estudo foi encaminhado ao Comitê de Ética e aprovado.

Jejum prolongado: foi realizado em 14 pacientes antes e em nove após o tratamento. Os pacientes foram mantidos em jejum por período de tempo variável, iniciando após o lanche noturno $(22 \mathrm{~h})$ ou pela manhã (8h). Durante o período do teste foram feitas coletas de sangue para dosagens plasmáticas de glicose, insulina e peptídeo-C, além da monitorização da glicemia com aparelhos de reflectância (Dextrometer, Glucometer I e II, Ames, USA). O jejum era suspenso quando os pacientes apresentavam sintomas evidentes de hipoglicemia e a glicemia capilar era inferior a $45 \mathrm{mg} / \mathrm{dL}$.

Teste do TOTG: foi realizado em quatorze pacientes antes do tratamento. Após coleta de sangue para dosagens plasmáticas basais de glicose e insulina, foi administrado $1,75 \mathrm{~g} / \mathrm{kg}$ de glicose por via oral (dose máxima $=75 \mathrm{~g}$ ), colhendo-se amostras após 30, 60, 90, 120,180 e 240 minutos.

Teste do Glucagon: foi realizado em 12 indivíduos pré-terapia e em quatro após esta. O Glucagon (Eli Lilly, Brasil) na dose de $1 \mathrm{mg}$ foi administrado via intravenosa (IV) após coleta de basais (glicose plasmática e insulina). As amostras após a infusão foram colhidas nos tempos $2,5,10,15,20,30,45,60,90$ e 120 minutos.

Os testes dinâmicos foram iniciados às $8 \mathrm{~h}$ da manhã após jejum noturno de $12 \mathrm{~h}$. A glicose plasmática foi determinada pelo teste enzimático colorimétrico (Biodiagnóstica, Brasil). A insulina foi mensurada por meio de radioimunoensaio (RIE) padronizado com anticorpos com $19 \%$ de reação cruzada com pró-insulina e 
0,003\% com peptídeo-C. A dosagem de peptídeo-C foi realizada por RIE com duplo anticorpo com estojo comercial da Immunonuclear (USA). O valor mínimo detectado foi de $0,1 \mathrm{ng} / \mathrm{mL}$ e havia $4 \%$ de reação cruzada com pró-insulina humana. Foram também colhidas amostras sanguíneas para dosagens de cálcio, fósforo, $\mathrm{PTH}$, gastrina, prolactina, hormônio de crescimento (hGH), antígeno carcinoembriônico, alfafetoproteína e $\beta$-HCG.

O estudo radiológico para localização dos tumores foi realizado por meio de ultrassonografia abdominal (Usab), endoscópica (Use) e intraoperatória (USio), tomografia computadorizada (TC) de abdome e ressonância nuclear magnética (RNM) de abdome. Em 13 pacientes, também foi realizada arteriografia pancreática com injeção de contraste no tronco celíaco para visualização de tumores. Venografia portal trans-hepática foi feita em nove pacientes, com localização da veia por- ta durante a fase venosa da arteriografia. Após a sedação dos pacientes, eram realizadas punção e cateterização da veia porta por via trans-hepática de acordo com a técnica de Lunderquist e Vang (1974). Foram realizadas determinações seriadas da concentração plasmática de glicose, insulina e peptídeo-C no pâncreas e simultaneamente na periferia para obtenção de gradientes.

O tratamento cirúrgico foi realizado em 22 pacientes (Tabela 1). Duas pacientes não foram operadas, uma tendo êxito letal após septicemia e a outra por não apresentar condições clínicas para cirurgia. O material da cirurgia foi submetido a exame anatomopatológico e em 10 pacientes também foi realizada imunoperoxidase pela técnica de avidina-biotina com incubação do soro com anticorpos primários para insulina (1/800-Dako), glucagon (1/150-Dako), cromogranina (1/500Boehringer-Mannheim), somatostatina (1/250-Dako), enolase (1/150-Dako) e gastrina (1/400-Dako).

Tabela 1. Características clínicas dos 24 pacientes com hipoglicemia por hiperinsulinismo endógeno e 0 tratamento realizado

\begin{tabular}{|c|c|c|c|c|c|}
\hline $\mathbf{N}^{0}$ & Idade (anos) & Sexo (M/F) & IMC $\left(\mathbf{k g} / \mathbf{m}^{2}\right)$ & $\begin{array}{c}\text { Duração da doença } \\
\text { (anos) }\end{array}$ & Tratamento \\
\hline 01 & 48 & M & 21,3 & 3 & EN \\
\hline 02 & 26 & M & 23,1 & 2 & $\mathrm{PCC}+\mathrm{EP}$ \\
\hline 03 & 51 & $\mathrm{~F}$ & 27,3 & 2,5 & $\mathrm{PCC}+\mathrm{EP}+\mathrm{EN}$ \\
\hline 04 & 16 & M & 22,5 & 2 & EN \\
\hline 05 & 68 & $\mathrm{~F}$ & 29,4 & 1 & $\mathrm{PCC}+\mathrm{EP}$ \\
\hline 06 & 17 & M & 13,6 & 0,3 & $\mathrm{PCC}+\mathrm{EP}$ \\
\hline 07 & 52 & $\mathrm{~F}$ & 27,9 & 14 & $\mathrm{PCC}+\mathrm{EP}$ \\
\hline 08 & 54 & M & 22,6 & - & PCC \\
\hline 09 & 13 & M & 23,3 & 1,5 & PCC \\
\hline 10 & 48 & $\mathrm{~F}$ & 30,7 & 3 & Óbito pré-tratamento \\
\hline $11^{*}$ & 38 & $\mathrm{~F}$ & 20,2 & 11 & $\mathrm{PCC}+\mathrm{EP}$ \\
\hline 12 & 20 & $\mathrm{~F}$ & 25,4 & 2,5 & $\mathrm{PCC}+\mathrm{EP}$ \\
\hline 13 & 66 & $\mathrm{~F}$ & - & 3 & $\mathrm{PCC}+\mathrm{EP}$ \\
\hline 14 & 73 & $\mathrm{~F}$ & 41,3 & - & Diazóxido \\
\hline 15 & 32 & $\mathrm{~F}$ & 34,8 & 8 & $\mathrm{PCC}+\mathrm{EP}$ \\
\hline 16 & 33 & $\mathrm{~F}$ & 27,6 & 8 & $\mathrm{PCC}+\mathrm{EP}$ \\
\hline 17 & 23 & $\mathrm{~F}$ & 23,0 & 0,75 & $\mathrm{EN}+\mathrm{M}$ \\
\hline 18 & 24 & M & 22,2 & 7 & PCC \\
\hline 19 & 39 & $\mathrm{~F}$ & 23,9 & 10 & PCC \\
\hline $20^{*}$ & 2,5 & M & 15,9 & 1,75 & PST \\
\hline 21 & 27 & $M$ & 23,0 & 1 & $\mathrm{PCC}+\mathrm{EP}$ \\
\hline 22 & 22 & $\mathrm{~F}$ & 27,6 & 5 & PCC+EP \\
\hline $23^{*}$ & 01 & F & 22,4 & 0,58 & PST \\
\hline 24 & 56 & $M$ & 25,3 & 1 & PCC \\
\hline $\mathrm{M} \pm \mathrm{DP}$ & $35,4 \pm 20,2$ & - & $25,0 \pm 5,8$ & $4,0 \pm 3,9$ & - \\
\hline
\end{tabular}

PCC: pancreatectomia corpo-caudal; EN: enucleação; PST: pancreatectomia subtotal; EP: esplenectomia; M: remoção de metástases; IMC: índice de massa corpórea.

* Casos de nesidioblastose. 
Análise estatística: as variáveis que apresentaram distribuição normal foram descritas como média \pm desvio-padrão e as variáveis que não apresentaram distribuição normal foram descritas como mediana (variação mínima e máxima). Para comparar variáveis com distribuição normal antes e depois do tratamento, foi utilizado o teste $t$ pareado. Para avaliar as variáveis que não apresentaram distribuição normal, foi utilizado o teste de Wilcoxon. Sensibilidade, especificidade e acurácia dos métodos diagnósticos foram calculados utilizando-se tabela de $2 \times 2$, em que $\mathrm{a}=$ verdadeiro positivo, $\mathrm{b}=$ falso-positivo, $\mathrm{c}$ $=$ falso-negativo e $\mathrm{d}=$ verdadeiro negativo. Sensibilidade $=\mathrm{a} /(\mathrm{a}+\mathrm{c})$, especificidade $=\mathrm{b} /(\mathrm{b}+\mathrm{d})$ e acurácia $=\mathrm{a}+\mathrm{d} /$ $(a+b+c+d)$. $O$ padrão-ouro de comparação estabelecido foi o histopatológico e a localização do tumor após a cirurgia. Os dados foram analisados por meio do software SigmaStat 1.0 (Jandel Scientific Corporation, Chicago, ilinois). Um $\mathrm{p}<0,05$ foi considerado significante.

\section{RESULTADOS}

\section{Sinais e sintomas}

Todos os pacientes apresentavam sinais e sintomas de hipoglicemia (adrenérgicos e ou neuroglicopênicos) que haviam iniciado em média 4,1 anos antes do diagnóstico. Entre os 24 indivíduos estudados, 15 (61\%) mostravam ambos os tipos de sintomas, $9(39 \%)$ apenas neuroglicopênicos e nenhum tinha somente manifestações adrenérgicas (Tabela 2).

Tabela 2. Manifestações clínicas dos 24 pacientes com hipoglicemia por hiperinsulinismo endógeno

\begin{tabular}{|c|c|}
\hline Sintomas & $\mathrm{N}^{0}$ de pacientes \\
\hline \multicolumn{2}{|l|}{ Neuroglicopênicos } \\
\hline Perda de consciência & $20(83,3 \%)$ \\
\hline Alteração de comportamento & $18(75,0 \%)$ \\
\hline Tontura & $12(50,0 \%)$ \\
\hline Convulsões & $10(41,6 \%)$ \\
\hline Cefaleia & $6(25,0 \%)$ \\
\hline Amnésia & $6(25,0 \%)$ \\
\hline Escotomas & $5(20,8 \%)$ \\
\hline Diplopia & $3(12,5 \%)$ \\
\hline \multicolumn{2}{|l|}{ Adrenérgicos } \\
\hline Sudorese & $11(45,8 \%)$ \\
\hline Tremores & $7(29,1 \%)$ \\
\hline Palidez & $7(29,1 \%)$ \\
\hline Palpitações & $3(12,5 \%)$ \\
\hline Extremidades frias & $1(4,1 \%)$ \\
\hline
\end{tabular}

O ganho de peso foi relatado por 9 (39\%) dos pacientes. O aparecimento dos sintomas foi predominante durante o jejum, pela manhã. Apenas um paciente apresentou queixas de hipoglicemia pós-prandial.

\section{Glicose, insulina e peptídeo-C após jejum de 12 horas}

Foram coletadas amostras em três dias consecutivos e considerada como valor diagnóstico a amostra com menor nível de glicose plasmática. Os valores pré e pós-tratamento de glicose, insulina e peptídeo-C são mostrados na tabela 3 e na figura 1 . Houve diferença entre os valores de glicose plasmática e insulina no pré e pós-tratamento (glicose $33 \pm 9,8 \mathrm{mg} / \mathrm{dL}$ vs. $109,7 \pm 67,3$ $\mathrm{mg} / \mathrm{dL}$ e insulina $92,4 \pm 174,2 \mathrm{uU} / \mathrm{mL} v \mathrm{~s} .16,7 \pm 19,7$ $\mathrm{uU} / \mathrm{ml}$, ambos $\mathrm{p}<0,0001)$. Houve claramente também uma tendência à queda nos valores de peptídeo-C no pré e pós-tratamento $(\mathrm{p}=0,07)$. Após o tratamento, todos apresentaram glicose plasmática de jejum $>70$ $\mathrm{mg} / \mathrm{dL}$, exceto um paciente que apresentou valor de $68 \mathrm{mg} / \mathrm{dL}$ após tratamento.

\section{Teste do jejum prolongado}

O tempo total de jejum variou de 7 a 47 horas $(20 \pm$ $12,9)$ (Figura 2). As dosagens plasmáticas no término do teste foram: glicose plasmática de $30,7 \pm 9,7 \mathrm{mg} / \mathrm{dL}$ (variando de 20 a $48 \mathrm{mg} / \mathrm{dL}$ ); insulina de 39,8 $\pm 25,3$ $\mathrm{uU} / \mathrm{mL}$ (variando de 2,5 a $79 \mathrm{uU} / \mathrm{ml}$ ) e peptídeo-C de $1,94 \pm 1,06 \mathrm{ng} / \mathrm{mL}$ (variando de 0,8 a $3,5 \mathrm{ng} / \mathrm{mL}$ ). A relação $\mathrm{I} / \mathrm{G}$ foi de $1,32 \pm 0,93$ (variando de 0,12 a $3,2)$. As concentrações de hGH e cortisol no final do jejum foram de $16,9 \pm 17,1 \mathrm{ng} / \mathrm{mL}$ (variando de 0,82 a $48,8 \mathrm{ng} / \mathrm{mL}$ ) e de $13,5 \pm 6,4 \mathrm{ng} / \mathrm{dL}$ (variando de 6,1 a $24,5 \mathrm{ng} / \mathrm{dL}$ ), respectivamente. Todos os pacientes apresentaram cetonúria negativa ao final do jejum.

\section{Teste oral de tolerância à glicose}

Entre os pacientes, sete já apresentavam hipoglicemia no basal, e apenas um não alcançou níveis normoglicêmicos durante o TOTG. Adicionalmente, apenas dois indivíduos tiveram glicose plasmática $\leq 50 \mathrm{mg} / \mathrm{dL}$ e outros dois, glicose $\leq 55 \mathrm{mg} / \mathrm{dL}$ nos tempos finais do teste (180' e 240'). Os demais pacientes não apresentaram hipoglicemia tardia após o teste.

\section{Teste do Glucagon}

Nos indivíduos antes do tratamento, os valores de glicose e insulina plasmáticas no tempo 0 ' foram de 43,9 
$\pm 13,6 \mathrm{mg} / \mathrm{dL}$ (variando de 28 a $72 \mathrm{mg} / \mathrm{dL}$ ) e de 39,8 $\pm 25,1 \mathrm{uU} / \mathrm{mL}$ (variando de 4 a $90 \mathrm{uU} / \mathrm{mL}$ ), respectivamente. A insulina teve seu pico máximo no tempo 5' de $271,8 \pm 350,5 \mathrm{uU} / \mathrm{mL}$ (variando de 12 a 1.036 $\mathrm{uU} / \mathrm{mL}$ ), decrescendo progressivamente até níveis de $28,6 \pm 17,7 \mathrm{uU} / \mathrm{mL}$ (variando de 4 a $59 \mathrm{uU} / \mathrm{mL}$ ) no tempo 120'. A glicose plasmática subiu até o tempo 20 ' para $87,1 \pm 32 \mathrm{mg} / \mathrm{dL}$ (variando de 27 a $129 \mathrm{mg}$ / $\mathrm{dL}$ ), ocorrendo uma queda posterior, com seus níveis mais baixos no tempo $120^{\prime}$ de 37,2 $\pm 10,1 \mathrm{mg} / \mathrm{dL}$ (variando de 21 a $52 \mathrm{mg} / \mathrm{dL}$ ). Adicionalmente, quatro pacientes realizaram o teste do Glucagon após o trata- mento (Figuras 3 e 4). Quando comparamos os níveis nos tempos 90' e 120' do teste do Glucagon antes e após tratamento, estes diferiram de maneira significante (glicose no tempo 90': $33,8 \pm 21,3 \mathrm{mg} / \mathrm{dL}$ vs. 88,3 $\pm 3,5 \mathrm{mg} / \mathrm{dL}, \mathrm{p}<0,05$; glicose no tempo 120': 43,9 \pm $13,6 \mathrm{mg} / \mathrm{dL}$ vs. $77 \pm 4,4 \mathrm{mg} / \mathrm{dl}, \mathrm{p}<0,0 \mathrm{l})$. Nenhum paciente apresentou hipoglicemia no pós-tratamento.

\section{Estudo radiológico}

Os nossos resultados são mostrados na tabela $4 \mathrm{em}$ comparação com outra série da literatura.

Tabela 3. Glicose plasmática, insulina e peptídeo-C dos 24 pacientes com hipoglicemia por hiperinsulinismo endógeno após jejum de 12 horas, no pré e pós-tratamento

\begin{tabular}{|c|c|c|c|c|c|c|c|c|}
\hline \multirow[b]{2}{*}{ № } & \multicolumn{4}{|c|}{ Pré-tratamento } & \multicolumn{4}{|c|}{ Pós-tratamento } \\
\hline & $\begin{array}{l}\text { Glicose } \\
\text { (mg/dL) }\end{array}$ & $\begin{array}{l}\text { Insulina } \\
\text { (uU/mL) }\end{array}$ & $I / G$ & $\mathbf{A l} / \mathbf{G}$ & $\begin{array}{l}\text { Peptídeo-C } \\
\text { (ng/mL) }\end{array}$ & $\begin{array}{l}\text { Glicose } \\
\text { (mg/dL) }\end{array}$ & $\begin{array}{c}\text { Insulina (uU/ } \\
\mathrm{mL})\end{array}$ & $\begin{array}{c}\text { Peptídeo-C } \\
\text { (ng/mL) }\end{array}$ \\
\hline 1 & 46 & 48 & 1,04 & 300 & - & 72 & 20 & - \\
\hline 2 & 27 & 14 & 0,51 & 466,7 & - & 91 & 8,6 & - \\
\hline 3 & 38 & 43 & 1,13 & 573,5 & 4,7 & 168 & 26 & 3,5 \\
\hline 4 & 32 & 36 & 1,12 & 1800 & - & 87 & 36 & 2,0 \\
\hline 5 & 45 & 29 & 0,64 & 193,3 & - & 98 & 12 & - \\
\hline 6 & 41 & 280 & 6,82 & 2545,4 & 1,17 & 73 & 4,1 & 0,48 \\
\hline 7 & 48 & 69 & 1,43 & 383,3 & 2,4 & 109 & 18,7 & 3,27 \\
\hline 8 & 29 & 18 & 0,62 & 1800 & - & 140 & 6,5 & - \\
\hline 9 & 24 & 123 & 5,12 & 2050 & 1,5 & 90 & 7,8 & - \\
\hline 10 & 27 & 105 & 3,9 & 3500 & 4,1 & - & - & - \\
\hline 11 & 22 & 100 & 4,54 & 1250 & - & 82 & 6,4 & - \\
\hline 12 & 37 & 76 & 2,05 & 1085,7 & 1,1 & 113 & 15 & 0,8 \\
\hline 13 & 40 & 135 & 3,37 & 1350 & - & 110 & 30 & - \\
\hline 14 & 28 & 87 & 3,1 & 4350 & - & 104 & 97,1 & - \\
\hline 15 & 43 & 20 & 0,46 & 153,8 & - & 109 & 14 & - \\
\hline 16 & 21 & 13 & 0,61 & 144,4 & - & 93 & 4,0 & - \\
\hline 17 & 18 & 860 & 47,7 & 7166,7 & 5,6 & 76 & 13 & 1,3 \\
\hline 18 & 49 & 18 & 0,36 & 94,7 & - & 108 & 2,2 & - \\
\hline 19 & 21 & 34 & 1,61 & 377,8 & - & 68 & 25 & 1,78 \\
\hline 20 & 28 & 12 & 0,42 & 600 & 0,6 & 77 & 4,4 & - \\
\hline 21 & 38 & 44,2 & 1,16 & 552,5 & 2,34 & 87 & 11,2 & 0,53 \\
\hline 22 & 39 & 18 & 0,46 & 200 & 2,29 & 78 & 5,1 & 0,34 \\
\hline 23 & 17 & 21,6 & 1,27 & 166,2 & - & 400 & 10 & - \\
\hline 24 & 33 & 13,1 & 0,39 & 436,6 & 4,2 & 90 & 6,5 & - \\
\hline$M \pm D P$ & $33^{*} \pm 9,8$ & $92,4^{\dagger} \pm 174,2$ & $3,7 \pm 9,5$ & $1314 \pm 1677,9$ & $2,7^{\ddagger} \pm 1,7$ & $109,7 \pm 67,3$ & $16,7 \pm 19,7$ & $1,6 \pm 1,2$ \\
\hline
\end{tabular}



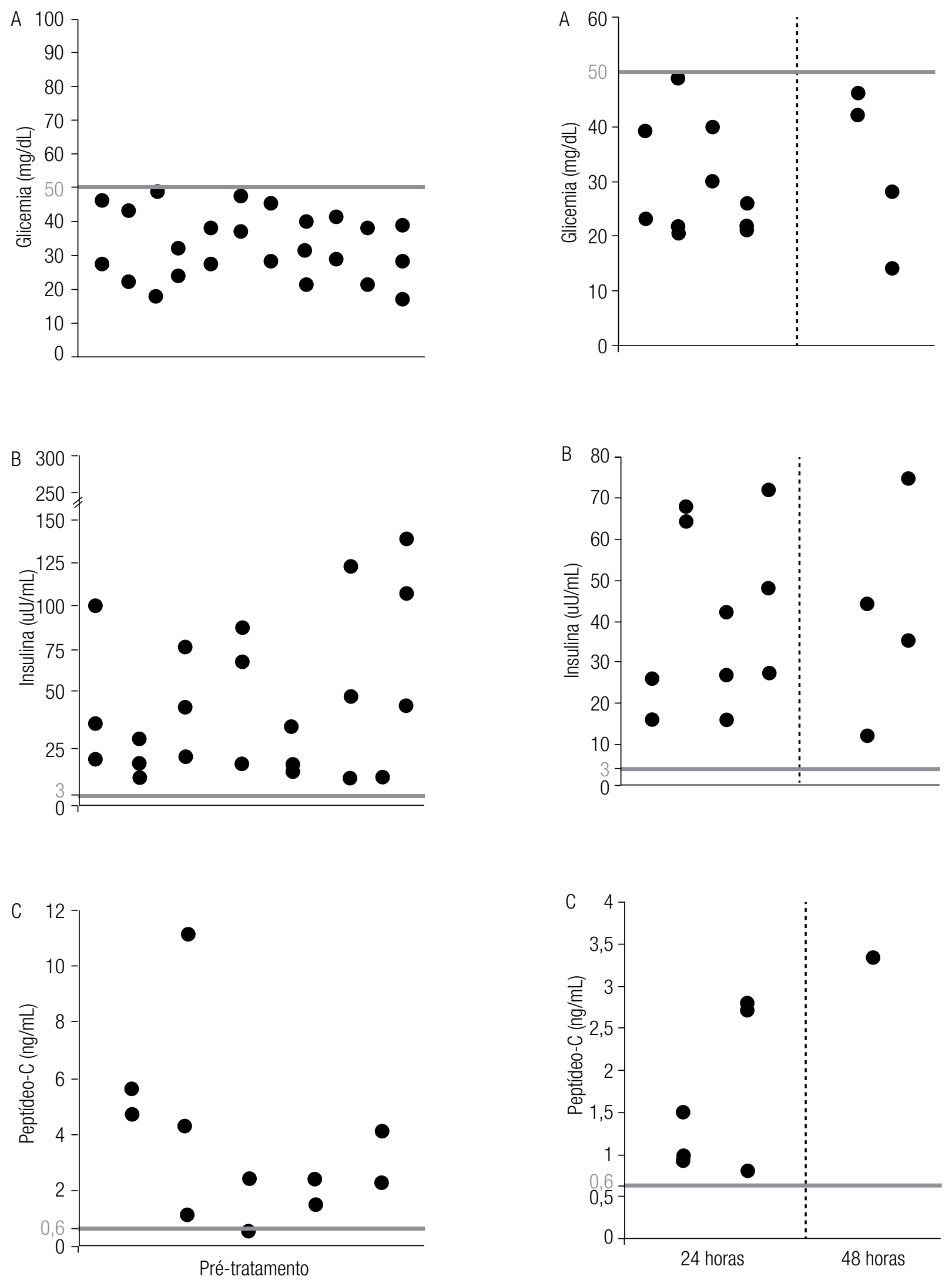

Figura 1. Teste do jejum de $12 \mathrm{~h}$ em 24 pacientes com hipoglicemia por hiperinsulinismo endógeno. Níveis de glicose plasmática (A), insulina plasmática (B) e peptídeo-C (C) antes do tratamento.

Figura 2. Teste do jejum prolongado em 14 pacientes com hiperinsulinismo endógeno. Níveis de glicose plasmática (A), insulina (B) e peptídeo-C (C) ao término do teste antes do tratamento. 

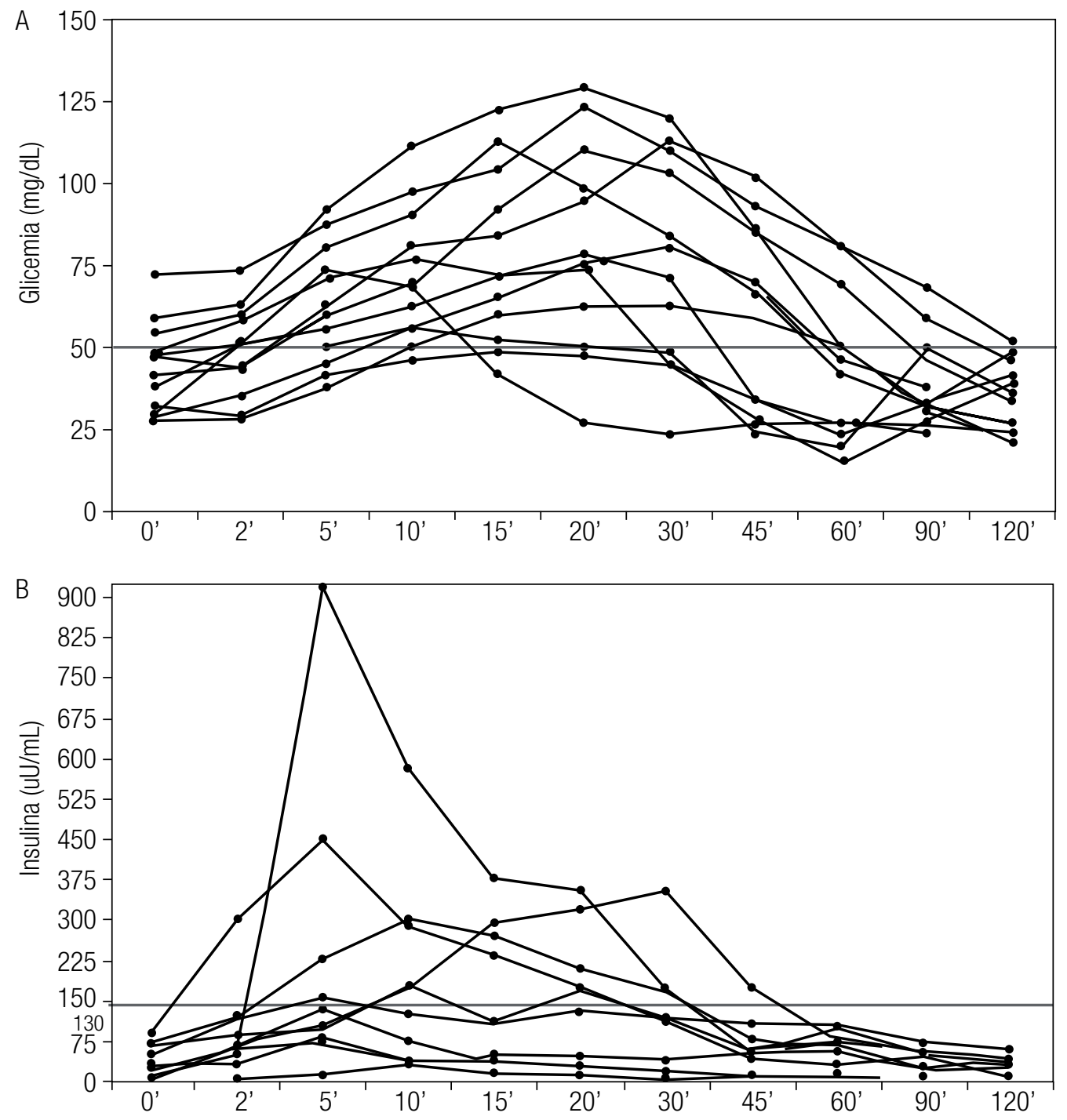

Figura 3. Teste do Glucagon com níveis de glicose plasmática (A) e insulina (B) em 12 pacientes antes do tratamento.

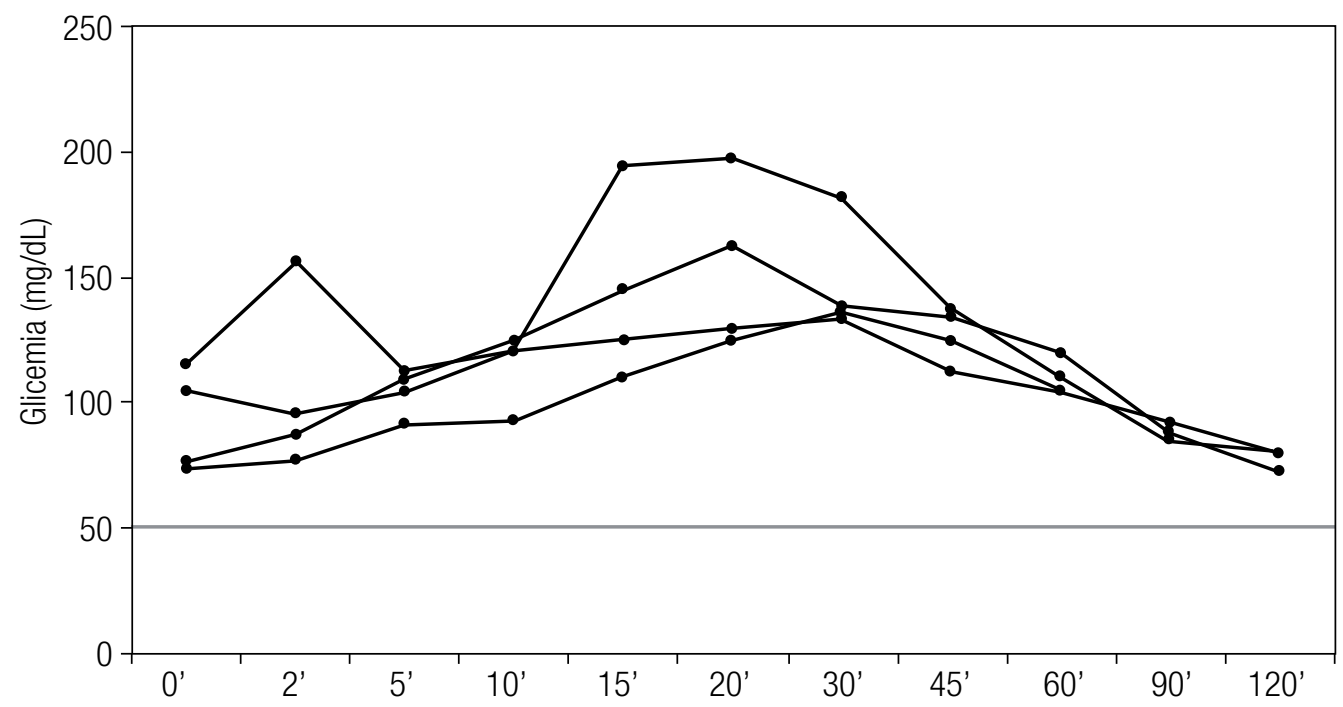

Figura 4. Teste do Glucagon com níveis de glicose plasmática em quatro pacientes após o tratamento. 
Tabela 4. Avaliação radiológica dos pacientes com diagnóstico de insulinoma

\begin{tabular}{lccccc}
\hline \multirow{2}{*}{ Método } & \multicolumn{2}{c}{ Felício e cols. } & & \multicolumn{2}{c}{ Liessi e cols. ${ }^{\mathbf{6 0}}$} \\
\cline { 2 - 3 } \cline { 5 - 6 } & $\begin{array}{c}\text { Total de } \\
\text { casos }\end{array}$ & Acurácia & & $\begin{array}{c}\text { Total de } \\
\text { casos }\end{array}$ & Acurácia \\
\hline Venografia & 9 & $9(100 \%)$ & & - \\
Arteriografia & 13 & $8(61,5 \%)$ & 6 & $3(50 \%)$ \\
TC de abdome & 17 & $2(11,7 \%)$ & 7 & $6(85,7 \%)$ \\
RNM de abdome & 2 & $1(50 \%)$ & 7 & $6(85,7 \%)$ \\
US de abdome & 17 & $7(41,2 \%)$ & 7 & $6(85,7 \%)$ \\
US intraoperatório & 4 & $4(100 \%)$ & 7 & $6(85,7 \%)$ \\
US endoscópico & 3 & $2(66,7 \%)$ & & - \\
\hline
\end{tabular}

RNM: ressonância nuclear magnética; TC: tomografia computadorizada; US: ultrassonografia.

\section{Tratamento}

A cirurgia foi realizada em 22 dos 24 pacientes com HHE. Em 17 (77,3\%) dos casos, optou-se por pancreatectomia corpo-caudal, dos quais 12 foram também submetidos à esplenectomia e um à enucleação. Em três indivíduos $(13,6 \%)$, realizou-se somente enucleação, e dois casos $(9,1 \%)$ portadores de nesidioblastose foram submetidos à pancreatectomia subtotal (Tabela 1). Entre os 19 pacientes portadores de insulinoma, 11 apresentavam adenoma único, cuja localização foi: cinco na cauda, quatro no corpo e dois na cabeça do pâncreas. Os oito restantes apresentavam adenomas múltiplos, sendo em seis destes estabelecido o diagnóstico de NEM-I. Uma paciente de 23 anos apresentava carcinoma das celulas- $\beta$. Complicações da cirurgia foram observadas em 13 pacientes, ocorrendo 11 casos de fístula pancreática e sete de abscesso intra-abdominal. Ambas as situações foram tratadas com antibioticoterapia e drenagem cirúrgica quando necessário, ocorrendo resolução em todos os casos. Quatro pacientes se tornaram diabéticos após a cirurgia. $\mathrm{O}$ tempo médio de seguimento dos pacientes após a cirurgia foi de 37 meses (variando de 1 a 160 meses). Uma criança com nesidioblastose precisou ser reoperada para normalização dos níveis glicêmicos. À exceção da paciente citada acima e do caso com carcinoma, nenhum outro paciente apresentou hipoglicemia após a primeira cirurgia. Entre os que continuam em seguimento em nosso serviço, não foi detectado nenhum caso de recidiva.

\section{Histopatológico e imunoperoxidase}

O exame histopatológico mostrou a presença de tumor de células- $\beta$ em 18 pacientes e nesidioblastose em três (sendo um caso em adulto). Realizou-se também imunoperoxidase em oito casos com insulinoma e em dois com nesidioblastose, cujos dados são mostrados na tabela 5 .
Tabela 5. Imunoperoxidase em oito pacientes com insulinoma e em dois com nesidioblastose (casos 20 e 23)

\begin{tabular}{lcccccc}
\hline Pacientes & Ins & Gluc & Crg & Enol & Smt & Gast \\
\hline 3 & +++ & NA & + & + & + & NA \\
6 & +++ & + & ++ & ++ & - & - \\
9 & +++ & + & NA & NA & NA & NA \\
12 & ++ & ++ & NA & + & + & +++ \\
17 & + & NA & + & + & NA & NA \\
18 & + & - & + & + & - & - \\
20 & ++ & + & NA & NA & + & NA \\
21 & +++ & - & NA & +++ & - & NA \\
22 & + & - & + & NA & + & - \\
23 & ++ & + & + & NA & + & NA \\
\hline
\end{tabular}

Ins: insulina; Gluc: glucagon; Crg: cromogranina; Enol: enolase; Smt: somatostatina; Gast: gastrina.

NA: não analisado; +: positivo focalmente; ++: positivo; +++: fortemente positivo; -: negativo.

\section{DISCUSSÃO}

Em nosso estudo foram avaliados os aspectos diagnósticos (clínico, laboratorial e radiológico) e o tratamento de 24 pacientes portadores de HHE decorrente de desordens das células- $\beta$ das ilhotas pancreáticas.

Entre os nossos casos, houve clara predominância dos sintomas neuroglicopênicos sobre os adrenérgicos. Em outras séries na literatura, tem ficado evidente o predomínio desse padrão em pacientes com insulinoma $(13,14)$. O ganho de peso é um achado comum (15) e foi observado em $39 \%$ dos pacientes deste estudo. Nosso grupo apresentou ainda $41,6 \%$ de casos de convulsões, os quais têm sido considerados uma manifestação incomum de hipoglicemia (16), exceto em crianças (17). Entretanto, na série da Mayo Clinic (13) foram encontradas convulsões do tipo grande mal em $12 \%$ dos casos.

A hipoglicemia é classicamente dividida em hipoglicemia pós-prandial ou reativa (até 5 h após a refeição) e pós-absortiva ou de jejum. Embora tenha sido muito utilizada no passado, essa classificação conforme a associação com o jejum não permite uma clara distinção etiológica, pois algumas desordens hipoglicêmicas podem apresentar ambos os padrões (10). A grande maioria dos pacientes de nosso estudo (22 de 23 ) apresentou os sintomas neuroglicopênicos durante o jejum, o que está de acordo com o padrão mais encontrado na literatura, independentemente da causa (18).

O diagnóstico de HHE é baseado na confirmação da hipoglicemia durante os sintomas, associada a elevados níveis de insulina. Assim, Service (19) estabeleceu 
como critérios diagnósticos níveis de insulina maiores que $6 \mathrm{mU} / \mathrm{mL}$ e de peptídeo-C maiores que 0,6 ng/ $\mathrm{mL}$, concomitantes com hipoglicemia sintomática de pelo menos $55 \mathrm{mg} / \mathrm{dL}$. Quando não for possível observar episódio espontâneo de hipoglicemia, é preciso intervir com o teste de jejum prolongado para demonstrar a hipoglicemia e determinar sua causa (20). Como alternativa, Frajans e Vinik (14), avaliando pacientes com HHE por desordens das células- $\beta$ pancreáticas, coletaram amostras após jejum de $12 \mathrm{~h}$ durante a noite por vários dias, tendo encontrado glicose plasmática $<50 \mathrm{mg} / \mathrm{dL}$ em $76 \%$ e glicose plasmática < $60 \mathrm{mg} /$ dL em $86 \%$ dos casos. Segundo esses autores, a taxa de sucesso desse teste (que eles denominam de minijejum) é baseada no fato de que $75 \%$ dos pacientes desenvolvem sintomas de hipoglicemia nas primeiras $18 \mathrm{~h}$ do jejum prolongado. Em nosso estudo, também foram realizadas coletas pela manhã, em três dias diferentes, após jejum de 12 horas durante a noite e foi possível diagnosticar HHE em 100\% dos pacientes (Figura 1). O mesmo procedimento foi feito por Vezzosi e cols. (21), sendo observada glicose plasmática $<60 \mathrm{mg} / \mathrm{dL}$ em $88 \%$ dos casos. Em seu estudo, eles concluíram que o teste do jejum prolongado continua sendo padrão-ouro no diagnóstico de HHE, apesar de terem defendido a criação de um critério diagnóstico bioquímico que seja capaz de evitar o teste do jejum prolongado, tido como desagradável e pouco tolerado por muitos pacientes, ou que pelo menos possa ser usado como triagem para excluir aqueles sem desordem hiperinsulinêmica.

É importante destacar que pessoas saudáveis podem apresentar níveis glicêmicos abaixo de $60 \mathrm{mg} / \mathrm{dL}$ ou mesmo abaixo de $45 \mathrm{mg} / \mathrm{dL}$ durante o teste do jejum prolongado (22), mas que raramente apresentam níveis $<70 \mathrm{mg} / \mathrm{dL}$ após $12 \mathrm{~h}$ de jejum durante a noite $(23)$. Merimee e Tyson (9) encontraram, em 40\% das mulheres magras saudáveis, que a glicose plasmática declina para níveis menores que $45 \mathrm{mg} / \mathrm{dL}$ durante o jejum de $72 \mathrm{~h}$, sendo que um terço apresentou valores entre $30 \mathrm{e}$ $35 \mathrm{mg} / \mathrm{dL}$. Hirshberg e cols. (24) questionaram a necessidade de estender o tempo do jejum para além das 48h. De fato, em nosso estudo, foi possível demonstrar a tríade de Whipple e diagnosticar HHE em até $48 \mathrm{~h}$ do teste do jejum prolongado em $100 \%$ dos pacientes (Figura 2). Adicionalmente, a experiência da Mayo Clinic em testes de jejum prolongados realizados em várias séries permitiu concluir que são necessárias as $72 \mathrm{~h}$ para que se possa diagnosticar os poucos casos de pacientes com desordens hipoglicêmicas que apresentam os crité- rios no terceiro dia do teste, evitando-se os falso-negativos ao máximo. Os resultados positivos para o teste do jejum prolongado em $48 \mathrm{~h}$ da Mayo Clinic foram $92 \%$ em 1976 (13) e 93\% em 2000 (20).

$\mathrm{Na}$ tentativa de resolver o problema dos falso-negativos, em série publicada por Service e cols. (25), pôde-se observar a importância do peptídeo-C no diagnóstico de HHE que, medido no final do jejum prolongado, obteve sensibilidade de $100 \%$ quando seu valor foi maior ou igual a $0,6 \mathrm{ng} / \mathrm{ml}$, concomitante à glicose plasmática menor ou igual a 50,4. Este foi capaz de separar todos os insulinomas dos indivíduos normais. Todos os nossos pacientes que foram submetidos ao jejum prolongado se enquadraram nesses critérios, confirmando os dados apresentados no estudo anterior.

Vezzosi e cols. (21) afirmam em seu estudo que a relação $\mathrm{I} / \mathrm{G}>0,3$, ou mesmo utilizando outros pontos de corte, não traz melhores resultados quanto à acurácia diagnóstica em relação à dosagem de insulina $\mathrm{e}$ peptídeo C por si só, e defendem que este último, em especial, possui maior acurácia, também, em relação à insulina. Apesar disso, nossos pacientes submetidos ao teste do jejum prolongado ou do jejum de $12 \mathrm{~h}$ apresentaram positividade para todos os critérios: glicose plasmática $<55 \mathrm{mg} / \mathrm{dL}$, insulina $>3 \mathrm{uU} / \mathrm{mL}$, peptídeo-C $>0,6 \mathrm{ng} / \mathrm{mL}$ e relação $\mathrm{I} / \mathrm{G}>0,3$. Caso seja necessário, o diagnóstico diferencial entre HHE e as outras hipoglicemias de jejum pode ser melhorado usando-se o "Amended I/G" (26).

Atualmente, novos ensaios específicos para dosagem de insulina e peptídeo C estão sendo usados, evitando a reação cruzada com pró-insulina e, com isso, diminuindo o ponto de corte da insulina para $3 \mathrm{mUI} / \mathrm{mL}$ $(3,22)$. Além disso, a dosagem de pró-insulina consiste em uma nova ferramenta diagnóstica cujos níveis acima de 5 pmol/L no momento da hipoglicemia (< $45 \mathrm{mg} /$ dL) são compatíveis com HHE, segundo Service (19).

Já foi demonstrado que pacientes com nesidioblastose podem apresentar teste de jejum prolongado de $72 \mathrm{~h}$ negativo e que o diagnóstico dessa desordem é mais difícil em relação ao insulinoma, sendo necessária, muitas vezes, a realização do TOTG de 5h (27). Raros são os casos de falso-negativo em pacientes com insulinoma relatados na literatura e cuja demonstração do estado hiperinsulinêmico e da hipoglicemia foi realizada mediante o TOTG de $5 \mathrm{~h}(28,29)$ ou do teste pós-refeição mista (3). Assim, muitos autores advogam o importante papel diagnóstico do TOTG de 5h para documentar a hipoglicemia nos casos de elevada suspei- 
ção de hiperinsulinismo, mas em que o teste do jejum foi negativo $(28,30)$. Por outro lado, o TOTG de $5 \mathrm{~h}$ também vem sendo muito criticado devido ao fato de que pelo menos $10 \%$ de indivíduos saudáveis apresentam um nadir de glicose plasmática $<50 \mathrm{mg} / \mathrm{dL}$ (3l) e, atualmente, ele vem sendo gradativamente substituído pelo teste pós-refeição mista na demonstração de hipoglicemia pós-prandial (3). Em nosso estudo, quatro pacientes apresentaram hipoglicemia nos tempos finais do teste (sendo que um deles tinha o diagnóstico de hiperplasia das células- $\beta$ ). Entretanto, não pudemos estabelecer a real importância do TOTG no diagnóstico do hiperinsulinismo endógeno, em decorrência de este ter sido realizado em pacientes com o teste do jejum prolongado positivo, o que caracteriza, por definição, a hipoglicemia de jejum (e não a reacional).

Conforme relatado anteriormente, nem todos os pacientes com HHE, durante o teste do jejum prolongado, apresentam níveis de glicose plasmática baixos o suficiente para o diagnóstico (32) e, segundo Vezzosi e cols. (21), pacientes com valores de glicose entre 45 e 60 podem se beneficiar com a dosagem seriada de $\beta$-hidroxibutirato, cuja concentração deve estar suprimida no HHE, sendo considerado um critério de boa acurácia para afastar essa condição. Isso se justifica, pois sua elevação durante o teste do jejum prolongado (lenta no início com posterior aumento acentuado na $30^{\mathrm{a}}$ hora) indica que, ao término, este será negativo, podendo também ser usado como um critério, quando maior que $2,7 \mathrm{mmol} / \mathrm{L}$, para finalizar o jejum antes das $72 \mathrm{~h}(18,33)$.

Portadores de insulinoma podem demonstrar uma resposta excessiva de insulina e subsequente hipoglicemia após injeção endovenosa (EV) de $1 \mathrm{mg}$ de glucagon (34). Em indivíduos normais, a glicose plasmática se eleva, atingindo o pico após 20 minutos, voltando aos níveis basais ao término do teste. A insulina atinge seu pico após 5 minutos do início deste, caindo gradativamente até o tempo de 120' (34). O limite superior do normal (Média + 2DP) para o pico de insulina é de $130 \mathrm{uUmL}(34,35)$. Alguns autores têm reportado que o teste do Glucagon é capaz de diagnosticar o insulinoma em 50 (5), 71 (34) e 72\% (12) dos casos. Em nossos pacientes, tomando por base este critério (pico de insulina > $130 \mathrm{uU} / \mathrm{mL}$ ), pudemos diagnosticar $54 \%$ dos insulinomas (Figura 3). Adicionalmente, encontramos em $11(91,7 \%)$ de 12 insulinomas níveis de glicose plasmática no tempo 120' menores que 50 $\mathrm{mg} / \mathrm{dL}$ e inferiores ao valor basal. O único paciente que não apresentou esse resultado teve glicose plasmática de $52 \mathrm{mg} / \mathrm{dL}$ ao final do teste, tendo partido de um basal de $47 \mathrm{mg} / \mathrm{dL}$ (Figura 3). Os pacientes que realizaram o teste após o tratamento não apresentaram hipoglicemia (Figura 4). É conhecido que outras causas de hipoglicemia (doença hepática, desnutrição etc.) podem apresentar só um pequeno aumento da glicose plasmática no início do teste e níveis hipoglicêmicos ao final deste $(34,36)$. Entretanto, nosso estudo sugere que a presença de níveis de glicose plasmática no tempo 120 ' do teste do glucagon inferiores a $50 \mathrm{mg} / \mathrm{dL}$ e ao valor basal reforça fortemente a hipótese de insulinoma. A administração de glucagon acrescenta ainda um importante dado ao final do teste de jejum prolongado: a elevação da glicose plasmática em pelo menos $25 \mathrm{mg} /$ $\mathrm{dL}$ após injeção EV de $\mathrm{l} \mathrm{mg}$ de glucagon indica que o estoque de glicogênio hepático está preservado e evidencia o excesso de insulina $(3,29,37)$.

Após a definição do quadro de HHE, o próximo passo é identificar a localização de um possível insulinoma. Mesmo em mãos experientes, técnicas radiológicas convencionais como ultrassonografia transabdominal, tomografia computadorizada e ressonância magnética podem não detectar o insulinoma. Entretanto, devido a alguns insulinomas apresentarem pequenas dimensões $(<1 \mathrm{~cm})$, imagens negativas não devem excluir o diagnóstico. A sensibilidade desses estudos radiológicos varia de $50 \%$ a $80 \%$. Quando a imagem não for localizada, o diagnóstico de nesidioblastose também deve ser considerado (38). Nossos resultados, quanto à limitação dos métodos citados, estão de acordo com os dados encontrados por outros autores $(39,40)$. A ultrassonografia endoscópica melhora a sensibilidade na detecção da imagem no pré-operatório para mais de 90\% $(41,42)$. Nossos resultados foram similares aos de Thompson e cols. (43), que obtiveram sucesso em $70 \%$ dos casos. Finalmente, a ultrassonografia intraoperatória tem sido considerada como o método mais sensível e específico, localizando a lesão com índices superiores a 95\%, principalmente combinada à palpação $(3,38,44)$. Em nosso estudo, a ultrassonografia intraoperatória foi capaz de localizar o insulinoma em 100\% dos casos (Tabela 4).

A venografia portal trans-hepática é um procedimento invasivo, porém apresenta alta sensibilidade (38). Os resultados da venografia portal são otimizados por meio da arteriografia pancreática seletiva com injeção de cálcio, uma vez que possibilita a regionalização exata do insulinoma (em 30\% a 85\% dos casos) quando as imagens convencionais são duvidosas ou negativas. 
Essa técnica é o procedimento de escolha na confirmação de HHE não insulinoma, cuja injeção de cálcio estimula a produção de insulina em todas as regiões do pâncreas, sugerindo o diagnóstico de nesidioblastose, na ausência da síndrome NEM-I, a qual apresenta frequentemente múltiplos insulinomas $(38,45-47)$. Essa técnica é importante também na decisão do tipo de tratamento a ser realizado. No nosso grupo, a venografia foi realizada em nove pacientes, apresentando acurácia de $100 \%$ e não ocorrendo complicações.

Há, ainda, a cintilografia com análogo da somatostatina (Octreoscan), cuja sensibilidade diagnóstica é de $60 \%$ a $80 \%$ para insulinomas $(48,49)$.

O estudo imuno-histoquímico dos insulinomas permite caracterizar melhor essas neoplasias, evidenciando que a maioria dos tumores endócrinos do pâncreas produz mais de um hormônio $(44,50,51)$. Na série de Apodaca-Torrez e cols. (44), verificou-se que $83,3 \%$ dos pacientes com diagnóstico clínico de insulinoma eram portadores de tumores produtores de dois ou mais hormônios, dos quais todos foram positivos para cromogranina. Nossa análise imuno-histoquímica apresentou positividade em $90 \%$ dos casos para pelo menos dois hormônios diferentes e todos também foram positivos para cromogranina, assim como para enolase. Embora a microscopia eletrônica ajude a diferenciar os tumores endócrinos pancreáticos pela presença de seus diferentes grânulos secretores, o diagnóstico de cada um deles depende da síndrome clínica concomitante (51). Assim, a imuno-histoquímica representa um método importante para o estudo dessas neoplasias (50).

A cirurgia é o tratamento de escolha para o insulinoma. Quando o tumor é único, a enucleação deve ser feita se possível. Na doença difusa das células- $\beta$, a maioria dos autores tem optado pela pancreatectomia subtotal (ressecção de $60 \%$ a $89 \%$ do pâncreas), que, segundo o estudo de Witteles e cols. (38), resulta em cura em cerca da metade dos casos, sem a necessidade de medicação, e normoglicemia em 19\% dos pacientes com uso de medicação no pós-operatório. Estudos sugerem que a pancreatectomia subtotal também deve ser indicada nos pacientes com NEM-I, mesmo quando somente um nódulo é localizado (52).

O tratamento clínico do insulinoma é baseado na dieta (14) e no uso de drogas hiperglicemiantes. Este é utilizado principalmente para controlar a glicose plasmática dos pacientes antes da cirurgia e naqueles que não têm condições clínicas de serem submetidos a esta. Entre as medicações usadas, destacam-se o diazóxido
$(4,5)$, de 6 a $15 \mathrm{mg} / \mathrm{kg} /$ dia divididos em 3 doses (53), e os análogos da somatostatina $(40,54,55)$. O último é considerado mais eficiente (14), sendo utilizado na dose de $3-8 \mathrm{mg} / \mathrm{kg} /$ dia em adultos e $10-30 \mathrm{mg} / \mathrm{kg} /$ dia em crianças $(56,57)$. Em nossa paciente com carcinoma das celulas- $\beta$, foi realizada quimioterapia com estreptozotocina e 5 -fluoracil após a cirurgia. Essa associação é considerada mais eficaz que a monoterapia com estreptozotocina $(58,59)$. A sobrevida dessa paciente foi de 55 meses, o que foi considerado satisfatório quando comparada à média de 26 meses encontrada na literatura $(58)$.

Em conclusão, foi encontrado em nosso estudo que a coleta de amostras de sangue após jejum de $12 \mathrm{~h} \mathrm{du}$ rante a noite em pelo menos três oportunidades pode tornar desnecessária, na grande maioria dos casos, a realização do jejum prolongado para o diagnóstico da hipoglicemia devido ao hiperinsulinismo. Confirmaram-se também os dados do estudo da Mayo Clinic, em que os valores de glicose plasmática e peptídeo- $\mathrm{C}$ ao final do jejum prolongado têm sensibilidade de cem por cento no diagnóstico do insulinoma. Finalmente, nosso estudo sugere que a presença de níveis de glicose plasmática no tempo 120' do teste do glucagon inferiores a $50 \mathrm{mg} / \mathrm{dL}$ e ao valor basal seja incluída como um critério adicional no diagnóstico dos tumores produtores de insulina, podendo este, eventualmente, ser utilizado para evitar a realização do jejum prolongado de $72 \mathrm{~h}$.

Declaração: os autores declaram não haver conflitos de interesse científico neste estudo.

\section{REFERÊNCIAS}

1. Cryer PE. Hypoglycemia, functional brain failure, and brain death. J Clin Invest. 2007;117:868-70.

2. Whipple AO. The surgical therapy of hyperinsulinism. J Int Chir. 1938;3:237-76.

3. Cryer PE, Axelrod L, Grossman AB, Heller SR, Montori VM, Seaquist $E R$, et al. Evaluation and management of adult hypoglycemic disorders: an Endocrine Society clinical practice guideline. J Clin Endocrinol Metab. 2009;94(3):709-28.

4. Marks V, Rose FC. Hypoglycemia. Oxford: Blackwell Scientific Publications; 1981 p. 51.

5. Service FJ. Hypoglycemic disorders. Boston: GK Hall Medical Publishers; 1983.

6. Haymond MW. Hypoglycemia in infants and children. Endocrinol Metab Clin North Am. 1999;18(1):211-52.

7. Cornblath M, Schwartz R. Disorders of carbohydrate metabolism in infancy. 2.ed. Philadelphia:WB Saunders Co; 1976.

8. Gastineau CF. Is reactive hypoglycemia a clinical entity? Mayo Clin Proc. 1983;58:545.

9. Merimee TJ, Tyson JE. Stabilization of plasma glucose during fasting. N Eng J Med. 1974;291:1275. 
10. Vella A, Service FJ. Incretin hypersecretion in post-gastric bypass hypoglycemia primary problem or red herring? J Clin Endocrinol Metab. 2007;92:4563-65.

11. Service FJ, McMahon MM, O'Brien PC, Ballard DJ. Functioning insulinoma - incidence, recurrence, and long-term survival of patients: a 60-year study. Mayo Clin Proc. 1991;66:711-9.

12. Stefanini P, Carboni M, Patrassi N, Basoli A. Hypoglycemia and insular hyperplasia: review of 148 cases. Ann Surg. 1974;180:130-5.

13. Service FJ, Dale AJD, Elveback LR, Jiang N-S. Insulinoma: clinical and diagnostic features of 60 consecutive cases. Mayo Clin Proc. 1976;51:417-29.

14. Frajans SS, Vinik Al. Insulin-producing islet cell tumors. Endocrinol Metab Clin North Am. 1989;18(1):45-74.

15. Dhume VM, Amarapurkar AD, Rege JD, Kandalkar BM, Varthakavi P. Insulinoma - A case report. Indian J Pathol Microbiol. 2004; 47:540-1.

16. Mulder DW, Rushton JG. Hyperinsulinism: rare cause of epilepsy. Neurology. 1959;9:288-9.

17. Hypoglycemia and the nervous system. Lancet. 1985;2:759-60.

18. Service FJ, Natt N. Clinical perspective: the prolonged fast. J Clin Endocrinol Metab. 2000;85:3973-4.

19. Service F. Hypoglycemic disorders. New Engl J Med. 1995;332:1144-52.

20. Johansen K. Insulinoma: clinical manifestations, diagnosis and treatment. The significance of the prolonged fasting test and of the fasting glucose-insulin relationship. J Endocrinol Invest. 1979;2:285.

21. Vezzosi D, Bennet A, Fauvel J, Caron P. Insulin, C-peptide and proinsulin for the biochemical diagnosis of hypoglycaemia related to endogenous hyperinsulinism. Eur $\mathrm{J}$ Endocrinol. 2007; 157:75-83.

22. Service F. Diagnostic approach to adults with hypoglycemic disorders. Endocrinol Metab Clin North Am. 1999;28:519-32.

23. Cryer PE. Clinical hypoglycemia. In: hypoglycemia pathophysiology, diagnosis and management. New York: Oxford University Press; 1997. p. 85-91.

24. Hirshberg B, Livi A, Bartlett DL, Libutti SK, Alexander HR, Doppman JL, et al. Forty-eight-hour fast: the diagnostic test for insulinoma. J Clin Endocrinol Metab. 2000;85:3222-6.

25. Service FJ, O'Brien PC, McMahon MM, Kao PC. C-peptide during the prolonged fast in insulinoma. J Clin Endocrinol Metab. 1993;76(3):655-9.

26. Hampton SM, Beyzavi K, Teale D, Marks V. A direct assay for proinsulin in plasma and its application in hypoglycemia. Clin Endocrinol (Oxf). 1988;29(1):9-16.

27. Starke A, Saddig C, Kirch B, Tschahargane C, Goretzki P. Islet hyperplasia in adults: challenge to preoperatively diagnose non-insulinoma pancreatogenic hypoglycemia syndrome. World J Surg. 2006;30:670-9.

28. Wiesli P, Schmid C, Perren A, PfammatterT, Spinas GA, Keller U. Hypoglycemia in response to glucose and glucagon in insulinoma patients with a negative prolonged fast: functional and morphological properties. J Endocrinol Invest. 2004;27:832-8.

29. Kar P, Price P, Sawers S, Bhattacharya S, Reznek RH, Grossman $A B$. Insulinomas may present with normoglycemia after prolonged fasting but glucose-stimulated hypoglycemia. J Clin Endocrinol Metab. 2006;91:4733-6.

30. KonngYG. Value of 5 -hour glucose tolerance test in the diagnosis of insulinoma. Zhonghua Wai Ke Za Zhi. 1990;28:331-2.

31. Hogan MJ, Service FJ, Sharbrough FW, Gerich JE. Oral glucose tolerance test compared with a mixed meal in the diagnosis of reactive hypoglycemia. A caveat on stimulation. Mayo Clin Proc. 1983;58:491-6.
32. Grant CS. Gastrointestinal endocrine tumours. Insulinoma. Baillieres Clin Gastroenterol. 1996;10:645-71.

33. Service FJ, O'Brien PC. Increasing serum betahydroxybutyrate concentrations during the 72-hour fast: evidence against hyperinsulinemic hypoglycemia. J Clin Endocrinol Metab. 2005;90:4555-8.

34. Marks V, Samols E. Glucagon test for insulinoma: a chemical study in 25 cases. J Clin Path. 1968;21:346-52.

35. Balcells A. Pruebas funcionales endocrinológicas. In: La clínica y el laboratorio: interpretación de ánalisis y pruebas funcionales exploración de los sindromes cuadro biológico de las enfermedades. Barcelona: MASSON; 2002. p. 418-9.

36. Field JB. Hypoglycemia. Endocrinol Metab Clin North Am. 1989;18(1):27-43.

37. Kaltsas GA, Besser GM, Grossman AB. The diagnosis and medical management of advanced neuroendocrine tumors. Endocr Rev. 2004;25:458-511.

38. Witteles RM, Straus II FH, Sugg SL, Koka MR, Costa EA, Kaplan EL. Adult-Onset nesidioblastosis causing hypoglycemia: an important clinical entity and continuing treatment dilemma. Arch Surg. 2001;136:656-63.

39. Doherty GM, Doppman JL, ShawkerTH, Miller DL, Eastman RC, Gorden $\mathrm{P}$, et al. Reults of a prospective strategy to diagnose, localize and resect insulinomas. Surgery. 1991;110:989-96.

40. Galiber AK, Reading CC, Charboneau JW, Sheedy PF 2nd, James EM, Gorman B, et al. Localization of pancreatic insulinoma: comparison of pre- and intraoperative US with TC and angiography. Radiology. 1988;166:405-8.

41. Fritscher-Ravens A. Endoscopic ultrasound and neuroendocrine tumours of the pancreas. JOP. 2004;5:273-81.

42. McLean AM, Fairclough PD. Endoscopic ultrasound in the localisation of pancreatic islet cell tumours. Best Pract Res Clin Endocrinol Metab. 2005;19:177-93.

43. Thompson NW, Czako PF, Fritts LL, Bude R, Bansal R, Nostrant TT, et al. Role of endoscopie ultrasonography in the localization of insulinomas and gastrinomas. Surgery. 1994;116:1131-8.

44. Apodaca-Torrez FR, Triviño T, Lobo EJ, Goldenberg A, Figueira A. Insulinomas do pâncreas: diagnóstico e tratamento. Arq Gastroenterol. 2003;40(2):73-9.

45. Brown CK, Bartlett DL, Doppman JL, Gorden P, Libutti SK, Fraker $\mathrm{DL}$, et al. Intraarterial calcium stimulation and intraoperative ultrasonography in the localization and resection of insulinomas. Surgery. 1997;122:1189-93.

46. Wiesli P, Brandle M, Schmid C, Krahenbuhl L, Furrer J, Keller U, et al. Selective arterial calcium stimulation and hepatic venous sampling in the evaluation of hyperinsulinemic hypoglycemia: potential and limitations. J Vasc Interv Radiol. 2004;15:1251-6.

47. Jackson JE. Angiography and arterial stimulation venous sampling in the localization of pancreatic neuroendocrine tumours. Best Pract Res Clin Endocrinol Metab. 2005;19:229-39.

48. Costa RR, Maia FFR, Araújo LR. Hipoglicemia hiperinsulinêmica persistente endógena no adulto: relato de caso. Arq Bras Endocrinol Metab. 2007;51(1):125-31.

49. Kumbasar B, Kamel IR, Tekes A, Eng J, Fishman EK, Wahl RL. Imaging of neuroendocrine tumors: accuracy of helical CT versus SRS. Abdom Imaging. 2004;29:696-702.

50. Mukai K, Greider MH, Grotting JC, Rosai J. Retrospective study of 77 pancreatic endocrine tumors using immunoperoxidase method. Am Surg Pathol. 1982;6:387-99.

51. Liu TH, Tseng HC, Zhu Y, Zhong SX, Chen J, Cui OC. Insulinoma: an immunocytochemical and morphologic analysis of 95 cases. Cancer. 1985;56:1420-9.

52. Demeure MJ, Klonoff DC, Karam JH, Duh OY, Clark OH. Insulinomas associated with multiple endocrine neoplasia type 1: the need for a different surgical approach. Surgery. 1991;110:998-1004. 
53. Ferraz DP, Almeida MAS, Mello BF. Uso de octreotide na hipoglicemia hiperinsulinêmica persistente infantil. Arq Bras Endocrinol Metab. 2005;49(3):460-7.

54. Alberts AS, Falkson G. Rapid reversal of life-threatening hypoglycemia with a somatostatin analogue (octreotide). S Afr Med J. 1988;74:75-6.

55. Hearn PR, Ahmed M, Woodhouse NJY.The use of SMS 201-995 in severe hyperinsulinemia. Diabetes Care 1988;11:664-8.

56. Grant DB, Dunger DB, Burns EC. Long term treatment with diazoxide in childhood hyperinsulinism. Acta Endocrinol (Suplemento) (Copenh). 1986;279:340-5.
57. Stanley CA, Baker L. Hyperinsulinism in infants and children: diagnosis and therapy. Adv Pediatr. 1976;23:315.

58. Moertel CG, Hanley JA, Johnson LA. Streptozocin alone compared with streptozocin plus fluorouracil in the treatment of advanced islet-cell carcinoma. N Engl J Med. 1980;303(21):1189-94.

59. Broder LE, Carter SK. Pancreatic islet cell carcinoma. Results of therapy with satreptozocin in 52 patients. Ann Intern Med. 1973;79:108-18.

60. Liessi G, Pasquali C, D’Andrea AA, Scandellari C, Pedrazzoli S. Magnetic resonance imaging in insulinomas: preliminary findings. Eur J Radiol. 1992;14:456-51. 